Cahiers Société

\title{
Idée de révolution et faire révolutionnaire chez Cornelius Castoriadis
}

\section{Benoît Coutu}

Numéro 1, mai 2019

Castoriadis et les sciences sociales

URI : https://id.erudit.org/iderudit/1068426ar

DOI : https://doi.org/10.7202/1068426ar

Aller au sommaire du numéro

\section{Éditeur(s)}

Collectif Société

ISSN

2562-5373 (imprimé)

2562-5381 (numérique)

Découvrir la revue

Citer cet article

Coutu, B. (2019). Idée de révolution et faire révolutionnaire chez Cornelius Castoriadis. Cahiers Société, (1), 193-205. https://doi.org/10.7202/1068426ar
Résumé de l'article

La philosophie politique de Cornelius Castoriadis est aujourd'hui réactualisée par nombre d'auteurs qui s'y réfèrent afin de jeter des bases théoriques à un renouveau des institutions politiques qui seraient plus aptes à faire face aux défis que nous impose le XXI ${ }^{\mathrm{e}}$ siècle. C'est dans ce contexte que nous proposons de revisiter sa théorie de la révolution. Pour ce faire, nous commencerons par la présentation de sa conception de la révolution, tant du point de vue historique que conceptuel. Nous exposerons par la suite les conditions objectives de possibilité qu'il met de l'avant, lesquelles sont agencées avec le désir comme conditions subjectives du passage à l'acte révolutionnaire. Nous verrons alors que celui-ci est pensé comme un faire dont le philosophe place en son centre la créativité dans et pour l'histoire. 


\title{
Idée de révolution et faire révolutionnaire chez Cornelius Castoriadis
}

\author{
Benoît COUTU \\ Université du Québec à Montréal
}

Nombre d'auteurs contemporains font référence à Cornelius Castoriadis dans leurs écrits appelant directement ou indirectement à une "révolution du $\mathrm{XXI}^{\mathrm{e}}$ siècle ». En général, ces références concernent ses thèses sur le politique et l'imaginaire, le social-historique, la praxis ou la pratique instituante, ou encore l'autofondation institutionnelle de la société. Elles esquivent les écrits dans lesquels le philosophe présente et développe ses idées sur ce qui est ou n'est pas, fut ou serait, une révolution, bien que sa théorie de la révolution ne se comprenne que par l'entremise de l'appareillage conceptuel mobilisé dans l'ensemble de son œuvre. Ce constat se présente alors à nous comme une invitation à explorer l'idée de révolution chez Castoriadis. À partir de quelques-unes de ses réflexions sur le sujet, nous présenterons comment il conçoit celle-ci, ce qui pourrait peut-être nous aider à penser la "révolution nécessaire " (dixit Jacques Ellul1) ou la "prochaine révolution » (dixit Léon Dion²) qui semble si peu encline à se réaliser. Pour ce faire, nous commencerons par exposer sa conception de ce qu'est ou n'est pas une révolution, tant d'un point de vue historique que conceptuel, touchant ainsi à l'exposition de ses conditions objectives de possibilité, mais aussi en filigrane à ce qui serait cause de blocage. Dans un second temps, nous aborderons la condition proprement subjective du passage à l'acte révolutionnaire. Ce passage par le désir nous permettra ensuite de développer plus en détail la question de l'agir révolutionnaire, en tant que la révolution est un faire - un faire « collectif, lucide et démocratique » - ce qui implique ultimement la reconnaissance de la centralité de la créativité dans et pour l'histoire.

\section{Une impossible révolution?}

Dans un entretien publié quelques années avant sa mort, Castoriadis se révélait plutôt sceptique face à la possibilité qu'advienne une révolution, ou du moins une révolu-

\footnotetext{
1 Jacques Ellul, «La révolution nécessaire ", dans Autopsie de la révolution, Paris, La Table Ronde, 2008, chap. 5, p. 273-352.

2 Léon Dion, La prochaine révolution, Montréal, Les Éditions Leméac, 1973.
} 
tion dite socialiste3, qui viendrait en quelque sorte parachever deux cents ans de révolution. En effet, en 1994, alors qu'une ère "nouvelle » s'annonçait au monde, celle de la globalisation, il avançait que le

mot « révolutionnaire » - comme les mots création ou imagination - est devenu un slogan publicitaire, c'est ce qu'on appelait il y a quelques années la récupération. La marginalité devient quelque chose de revendiqué et de central, la subversion est une curiosité intéressante qui complète l'harmonie du système. Il y a une capacité terrible de la société contemporaine à étouffer toute véritable divergence, soit en la taisant, soit en en faisant un phénomène parmi d'autres, commercialisé comme les autres4.

Cet enlisement du projet révolutionnaire, tant sur le plan des conditions objectives que des conditions subjectives, Castoriadis l'avait déjà pressenti dès la fin des années 1950 et cette interrogation s'échelonnera en trois moments au courant de sa vie. Premièrement, dans une critique du marxisme traditionnel qu'il réalise à cette époque, il dénote une "absence du prolétariat à la société5》, appelant le mouvement révolutionnaire à réactualiser la théorie critique de la société afin d'élucider le lien entre modernisation et dépolitisation des masses, ce qui pour lui constituait alors le point de départ d'une nouvelle politique révolutionnaire qui prendrait en compte l'évolution du capitalisme ${ }^{6}$. À cette occasion, Castoriadis dénonça aussi les organisations politiques ouvrières professionnalisées et bureaucratisées, l'attitude des classes populaires réduites à de simples classes en soi définies par les rapports de production qui les conçoivent, ainsi que le blocage constitué par le développement exponentiel de la technique.

Par la suite, à la fin des années 1970, donc après Mai 68 et l'émergence des nouveaux mouvements sociaux, il ajoutera à sa critique initiale, principalement dirigée contre le marxisme orthodoxe, le fait que l'action collective ne concerne plus que «d'infimes minorités» sans bouleverser le cours de la société7. Celle-ci n'est d'ailleurs plus remise en question, entre autres en raison de la domination de la pensée par la pure spéculation philosophique et le déconstructivisme, lesquels parachèvent ce qu'il nomme la "privatisation des individus» - un individu qui, replié

\footnotetext{
3 Il conçoit qu'une révolution peut être de «droite » ou étatique, entre autres sous la forme du néoconservatisme.

4 Olivier Morel, «Un monde à venir - entretien avec Cornelius Castoriadis », 1994. En ligne : http://1libertaire.free.fr/castoriadis06.html

5 Cornelius Castoriadis, «Le mouvement révolutionnaire sous le capitalisme moderne » [1959], dans Capitalisme moderne et révolution, II. La mouvement révolutionnaire dans le capitalisme moderne, Paris, Union Général d'Éditions, 1979, p. 52.

6 Ibid., p. 59 sq.

7 Impossible d'y arriver par des changements «millimétriques ». Entretien avec Cornelius Castoriadis, «L'idée de révolution a-t-elle encore un sens ? », Le Débat, vol. 5, n 57, 1989, p. 220.
} 
sur lui-même et lové dans sa jouissance, a délaissé la passion pour les affaires communes.

Enfin, dans les années 1990, la cristallisation de ces faits lui semble aboutir à la réduction des possibilités d'une révolution, caractérisant de ce fait la fin du $\mathrm{XX}^{\mathrm{e}}$ siècle par «[1]'effondrement graduel puis accéléré des idéologies de gauche, le triomphe de la société de consommation, la crise des significations imaginaires de la société moderne [significations de progrès et/ou de révolution ${ }^{8}$ ]». Surtout, cette crise portée par ces trois moments du triomphe du capitalisme, manifeste à ses yeux « [...] une crise du sens et c'est cette crise du sens qui permet aux éléments conjoncturels de jouer le rôle qu'ils jouent 9 ».

\section{L’idée de révolution}

Ce qui perd de son sens dans tout ce brouhaha, c'est la vie elle-même, bien sûr, en raison d'un décalage abyssal entre les structures sociales objectives et les conditions subjectives des individus ou, pour le dire dans les termes de Charles Wright Mills, de l'écart gigantesque entre l'historicité de la société et la biographie des individus qui ne s'y reconnaissent plus ${ }^{10}$. Castoriadis décrit la situation comme un abîme entre le social-historique (qui tend à disparaître) et les significations imaginaires (porteuses du projet révolutionnaire). Mais, dans ce désancrage, nous avons aussi perdu le sens de l'idée de révolution, comme il l'appelle. Castoriadis a traité de ce sujet lors d'une entrevue intitulée «L'idée de révolution a-t-elle encore un sens ${ }^{11}$ ? », publiée dans le cadre des festivités entourant le $200^{\mathrm{e}}$ anniversaire de la Révolution française. Pour l'occasion, il est demandé à Castoriadis de «situer la Révolution française dans la série des grandes révolutions" modernes. Bien que l'objectif soit de présenter la spécificité de cette révolution, le philosophe en profite pour poser les jalons de toute réflexion critique et contemporaine sur l'idée de révolution. En qualifiant la Révolution française de «première révolution qui pose clairement l'idée d'une autoinstitution explicite de la société12 », Castoriadis s'inscrit dans une longue lignée d'auteur.e.s qui tentent de cerner sociologiquement le concept de révolution en établissant une distinction conceptuelle entre des révolutions sociales et des révolutions politiques, les premières étant de plus grande envergure que les secondes, à ce point que plusieurs parlent de révolution que dans le cadre de la première, les secondes étant associées à des réformes, des révoltes ou des changements de régime qui ne remettent en question ni l'ordre social ni le mode de production existant, et

\footnotetext{
8 Olivier Morel, « Un monde à venir », op. cit., p. 4.

9 Idem.

10 Charles Wright Mills, L'imagination sociologique, Paris, La Découverte \& Syros, 1997, p. 5-7.

${ }^{11}$ Cornelius Castoriadis, «L'idée de révolution a-t-elle encore un sens ? », op. cit.

12 Ibid., p. 215.
} 
n'impliquant pas de prise de conscience globale ${ }^{13}$. Nous en déduisons donc que pour Castoriadis, ce qui fait la spécificité d'une révolution, en comparaison à ce qu'il nomme des « ré-institutions », est qu'elle est, en partant, une «entreprise d'autoinstitution explicite » qui fait rupture avec le passé.

Ainsi, la première appréhension de Castoriadis est, pourrait-on dire, ontologique et historique : une vraie révolution au sens moderne du terme est une prise en charge du peuple par lui-même dans un mouvement de création du nouveau qui doit mener à son autonomie, et ainsi dépasser une situation qu'il qualifie d' « infantile ». Historiquement, du moins jusqu'au début de la Révolution française, la révolution était conçue comme un retour à une autorité perdue, à un âge d'or utopique corrompu au fil des années, bref à l'idée de révolution, telle qu'elle était perçue depuis Platon jusqu'à Hobbes et Rousseau ${ }^{14}$, comme le recommencement d'un cycle. Il est évident, dit Castoriadis, que les Grecs connaissaient l'auto-institution de la société, qui se réalisait à partir du nomos, mais cela n'est pas pour lui le « principe de l'activation politique couvrant la totalité de l'institution sociale », puisque la propriété, le statut des femmes ou l'esclavage n'ont jamais été remis en question ${ }^{15}$.

Par ailleurs, il rappelle que l'Américain Thomas Jefferson était contre l'industrialisation, la voyant comme une menace à la libre propriété agraire, base de la liberté politique selon lui. Dès lors, aux yeux de Castoriadis, allant ici à l'encontre de Hannah Arendt ${ }^{16}$, la révolution américaine n'avait pour but que de préserver un ordre social ancien, en ce qu'il s'agissait davantage de donner une structure politique en continuité avec le passé que de produire un ordre social nouveau. C'est dire alors que ce qui fait la spécificité de la Révolution française - faire rupture et instituer du nouveau - ne se retrouve pas dans les révolutions anglaise et américaine. En restant " ancrée dans le religieux » (forme américaine) ou dans le passé (par la Common Law pour la révolution anglaise), ou les deux en même temps, elles se limitèrent à « rétablir une harmonie sociale jugée perdue ${ }^{17}$ ». Ainsi, lorsqu'il affirme que la grandeur et l'originalité de la Révolution française tient au fait qu'elle « tend à mettre en question, en droit, la totalité de l'institution existante de la société18 », cela implique

13 Benoît Coutu, «Bilan de campagne suivi de Révolution : borne, chemin et pli de la sociologie », dans Révolutions et contre(-)pouvoirs : réflexions sur l'agir politique en des temps incertains, dir. Benoît Coutu, Montréal, Les Éditions libres du carré rouge, 2017, p. 9-21.

14 Raymond Williams, Keywords, London, Flamingo Edition, 1981, p. 270-274.

15 Cornelius Castoriadis, «L’idée de révolution a-t-elle encore un sens ? », op. cit., p. 214.

16 Ce n'est pas le seul point de désaccord entre Castoriadis et Arendt. Un peu plus loin, il avance que « Hannah Arendt commet une bévue énorme lorsqu'elle reproche aux révolutionnaires français de s'être occupés de la question sociale. [...] D'abord [...] la question sociale est une question politique. [...] Ensuite, en France l'Ancien Régime n'est pas une structure simplement politique ; c'est une structure sociale. [...] C'est tout l'édifice qui est à reconstruire, sans quoi une transformation politique est matériellement impossible». Pour conclure avec ce commentaire vitriolique : "Comme si souvent chez Hannah Arendt, les idées empêchent de voir les faits ». Ibid., p. 214-215.

17 Idem.

18 Idem. 
autant la distinction entre révolution et réforme (ou révolte) que la distinction entre ce que les auteur.e.s issus du marxisme anglo-saxon contemporain entendent par « révolution sociale » et « révolution politique ». Mais au contraire de ceux-ci qui ne font que les différencier, Castoriadis les articule. En effet, selon lui, une véritable révolution implique nécessairement un processus de destruction-création, destruction de l'ordre ancien et reconstruction de « tout l'édifice social » sans lequel « une transformation politique est matériellement impossible ${ }^{19}$ », faisant dès lors de la première une condition de la seconde. Autrement dit, on ne peut superposer une nouvelle organisation politique sur un ordre social ancien, d'où les écueils de la révolution américaine : tenter de fonder un système postulant l'égalité sociale sur un ordre social inégalitaire. Ainsi, à la différence d'une révolte, Castoriadis définira la révolution comme :

des moments où la masse $[. .$.$] non seulement « se révolte » contre$ l'ancien ordre, mais veut modifier les institutions sociales de fond en comble [...]. Ce sont des révolutions, parce que les gens sont animés par une volonté et une visée globales ${ }^{20}$.

En bref, nous pourrions dire que la révolution se fait par le bas (par le «peuple») et non par le haut (à partir des volontés d'un État), et c'est d'ailleurs pour cette raison que Castoriadis est cité par Dardot et Laval dans leur ouvrage sur la révolution au $\mathrm{XXI}^{\mathrm{e}}$ siècle ${ }^{21}$. L'idée centrale est donc celle-ci : pour qu'elle soit nommée et reconnue comme telle, il faut que la révolution réalise «l'auto-institution explicite de la société par l'activité collective, lucide et démocratique $22 »$. Trois critères donc : collective, lucide et démocratique, lesquels se rencontrent dans la praxis - dans le faire $\mathrm{du}$ projet révolutionnaire. "Le monde historique [étant] le monde du faire humain $23 »$, il faut comprendre alors que la

révolution ne signifie pas seulement tentative de ré-institution explicite de la société. La révolution est cette ré-institution par l'activité collective et autonome du peuple, ou d'une grande partie de la société24.

Toujours est-il que cette activité doit prendre un caractère démocratique, et la lucidité s'insère ici, via la praxis, comme médiation entre l'activité collective et la volonté démocratique. En effet, dit-il, ce caractère démocratique est impossible à concevoir si les révolutionnaires modernes restent obnubilés par le fantasme d'une

\footnotetext{
19 Ibid., p. 214-215.

20 Olivier Mongin et Cornelius Castoriadis, «L'exigence révolutionnaire. Entretien avec Cornelius Castoriadis », Esprit, vol. 2, n 2, fév. 1977, p. 217.

${ }^{21}$ Pierre Dardot et Christian Laval, Commun. Essai sur la révolution au XXI siècle, Paris, La Découverte, 2016.

22 Cornelius Castoriadis, «L'idée de révolution a-t-elle encore un sens ? », op. cit., p. 216.

23 Cornelius Castoriadis, L'institution imaginaire de la société, Paris, Seuil, 1999, p. 106.

24 Cornelius Castoriadis, «L'idée de révolution a-t-elle encore un sens ? », op. cit., p. 219.
} 
maîtrise rationnelle de l'histoire et de la société, laquelle est une caractéristique du totalitarisme selon Castoriadis. D'un autre côté, rester pris dans le piège de la " révolution pour la révolution », de la rupture pour la rupture, n'est pas plus révolutionnaire. Au contraire, "le problème d'une révolution est d'instaurer un autre rapport à la tradition $25 »$. Si la révolution est rupture avec le passé, elle ne signifie pas négation du passé, puisque cette rupture n'est pas pour le philosophe une créationex nihilo. Cette rupture, dit-il, se situe «dans le sens nouveau qu'elle confère à ce qu'elle hérite ou utilise 26 ». D'où émane ce sens nouveau ? De l'activité, du « travail du faire », de la praxis - qui est la médiation entre le collectif, la lucidité et la démocratie.

Un des premiers actes de lucidité est de nous rendre compte que nous vivons non au sein de démocraties, mais sous des régimes d'oligarchie libérale qui reconduisent la division entre "pouvoir et non-pouvoir », qu'il faudrait abolir. De cette situation résulte que la société d'aujourd'hui « produit essentiellement des avides, des frustrés et des conformistes $27 »$. En éloignant les citoyens des affaires publiques, les institutions politiques actuelles créent de l'apathie et une dépolitisation des masses, doublement entretenues par la domination des médias et par ce qu'il nomme des «politiciens en pellicule 28 ». Empêchant l'activité politique, le problème de la démocratie actuelle est qu' «il n'y a plus de citoyens, il y a des consommateurs qui se contentent d'un vote d'approbation ou de désapprobation tous les cinq ans 29 ». Et quand la «passion pour les affaires communes [ne se transmet, ne se transforme plus en] affaires personnelles de chacun 30 », il n'y a plus de remises en question fondamentales, ni de radicalisme politique. Il ne leur reste alors que « la garantie de [leurs] jouissances », une formule qu'il reprend à Benjamin Constant31.

Si ce thème de la jouissance revient constamment dans ses écrits sur la révolution, c'est qu'il oblige à réfléchir au type d'homme correspondant à la société contemporaine, et inversement. C'est donc dans l'articulation organique entre la structure psycho-sociale et les institutions démocratiques que la pensée de Castoriadis se déploie. Pour Castoriadis, « [à] toute institution correspond un type anthropologique qui en est le porteur concret [...] et à la fois son produit et la condition de sa reproduction 32 ». Or, c'est le type d'homme démocratique, au jugement indépendant et animé par les questions de portée générale qui est aujourd'hui remis en cause33, remplacé par un " individu privé ». Cette idée de la jouissance "privée ou privative » est en

25 Idem.

26 Ibid., p. 215.

27 Ibid., p. 220.

28 Ibid., p. 221.

29 Ibid., p. 223.

30 Idem.

31 Ibid., p. 221.

32 Ibid., p. 222.

33 Idem. 
phase avec l'idée que l'économie est la valeur centrale de la société contemporaine : «Derrière les jouissances [...] il y a l'économie : les "jouissances" sont la face subjective de ce qu'est devenue l'économie dans le monde moderne, c'est-à-dire la "réalité" centrale, la chose qui compte vraiment $34 »$. Déchirées entre ces deux pôles, la jouissance et les valeurs déterminées par l'économie, s'immiscent «tout au plus, quelques petites libertés [...] tolérées comme complément instrumental du dispositif maximisateur35 ». Avec l'évanescence de la liberté disparaît l'autonomie, car qu'estce qu'une société libre sinon une société autonome dans laquelle les individus libres et autonomes participent collectivement à l'élucidation, et donc à l'auto-institution d'une nouvelle société égalitaire?

[...] Une société qui se donne à elle-même, effectivement et relativement, ses propres lois, sachant qu'elle le fait. Qu'est-ce qu'un individu libre, ou autonome, du moment où il n'est concevable que dans une société où il y a des lois et du pouvoir ? C'est un individu qui reconnaît dans ces lois et ce pouvoir ses propres lois et son propre pouvoir - ce qui ne peut se faire sans mystification que dans la mesure où il a la pleine possibilité effective de participer à la formation des lois et à l'exercice du pouvoir36.

\section{Du désir au faire}

Il nous faut, rendu ici, penser l'articulation entre le désir et le faire, ce qui appelle à interroger les « racines subjectives du projet révolutionnaire». Quelles sont-elles ? Ce sont les motivations et les attitudes capables de mener le projet révolutionnaire à son terme. Castoriadis écrit : « un révolutionnaire ne peut pas poser des limites à son désir de lucidité37». La lucidité, c'est vouloir une autre société, avoir le désir d'une autre société. «[U]ne action véritable, affirme-t-il, une praxis, implique le pouvoirêtre autrement de ce qui est dans sa profondeur, comme signification et comme valeur - et bien entendu aussi le vouloir-être-autrement 38 . " Ce désir d'être autrement est dans un premier temps de l'ordre d'un ressentir: "je sens le besoin», dit Castoriadis39. Mais ce ressentir n'est pas une totalité close, elle inclut nécessairement l'autre, c'est un désir partagé, en mouvement vers l'autre :

\footnotetext{
34 Ibid., p. 222-223.

35 Ibid., p. 223.

36 Ibid., p. 220.

37 Cornelius Castoriadis, L'institution imaginaire de la société, op. cit., p. 135.

38 Olivier Mongin et Cornelius Castoriadis, «L'exigence révolutionnaire. Entretien avec Cornelius Castoriadis », op. cit., p. 203.

39 Cornelius Castoriadis, L'institution imaginaire de la société, op. cit., p. 136.
} 
Je désire qu'autrui soit libre, car ma liberté commence là où commence la liberté de l'autre et que, tout seul, je ne peux être au mieux que « vertueux dans le malheur 40 ».

Ce désir n'est donc pas une pure illusion subjective ou une recherche de satisfaction personnelle, mais est de l'ordre du collectif. Et puisqu'il est un rapport social avant d'être un rapport à soi, le désir doit être travaillé, en commun, ce qui impose de faire des choix, et c'est dans ce travail en commun qu'advient un sens nouveau, produit tout autant que producteur du projet révolutionnaire. Le sens advient donc de la participation à une collectivité de révolutionnaires, il est inscrit dans la pratique. La liberté et l'autonomie ne peuvent émaner que d'une pratique collective. Ultimement, pour Castoriadis, ce désir se concrétise dans l'abolition du pouvoir au sens actuel, ce pouvoir qui postule que les autres sont des choses, et qui fait que je suis moi-même une chose : « Je veux la suppression du Guignol et la transformation des hommes en pantins par d'autres pantins qui "gouvernent 41 " ».

Ce désir qui se transforme en faire repose sur la volonté que « la Loi ne me soit pas simplement donnée, mais que je me la donne en même temps à moi-même ${ }^{42}$ ». Cette condition d'autonomie, Castoriadis la retrouve dans la révolution socialiste, car elle «vise la transformation de la société par l'action autonome des hommes, et l'instauration d'une société organisée en vue de l'autonomie de tous 43 ». Ce n'est pas une théorie, précise-t-il, c'est un projet, un projet qui prend ses racines et ses points d'appui dans la réalité historique effective, qui est celle de «la crise de la société établie et sa contestation par la grande majorité des hommes qui y vivent 44 ».

Pour réaliser ce projet de transformation sociétale, il faut commencer par se débarrasser de l'idée d'une dialectique totale ou d'une histoire totale, laquelle empêche de concevoir qu'il y a plusieurs possibilités, plusieurs horizons45. Concevoir qu'il y a plusieurs possibilités, que l'histoire est ouverture, suppose qu'on ne part pas du vide, mais toujours de quelque part et d'une perspective particulière. Toutefois, le projet révolutionnaire ne tient pas de l'arbitraire subjectif de quelques individus, tout comme la crise n'est pas une crise en soi, mais est une crise du fait qu'elle est en même temps contestation, qu'elle émane d'une contestation et la nourrit constamment. Les mouvements de contestation prennent forme autour «de faits lourds de sens» (« usure de l'autorité, épuisement des motivations économiques, atténuation de

\footnotetext{
40 Ibid., p. 138.

41 Ibid., p. 140.

42 Ibid., p. 139.

43 Ibid., p. 130.

44 Ibid., p. 141.

45 Contre la «dialectique totale ou l'histoire totale », Castoriadis soutient qu'il y a toujours plusieurs possibilités, plusieurs horizons : «l'histoire totale, de partout et nulle part, c'est le fait d'une pensée sans horizon, qui n'est qu'un autre nom de la non-pensée ». Ibid., p. 150.
} 
l'emprise de l'imaginaire institué, non-acceptation des règles héritées ${ }^{46} \gg$ ) organisés autour de significations centrales : d'un côté la fragmentation sociale et le repli sur soi, de l'autre la coopération et l'autogestion collective. C'est dans «l'ensemble de ces phénomènes [que] surgiss[ent] la possibilité et la demande d'autonomie dans la société47 ». Dès lors, pense-t-il, le socialisme est la voie, car la visée d'autonomie promulguée et soutenue qui s'y rattache s'insère dans l'histoire passée : c'est un projet qui naît de l'histoire dans laquelle «nous [...] reconnaissons nos aspirations les plus profondes, et nous pensons que sa réalisation est possible 48 ».

Nous le voyons, pour Castoriadis, le projet révolutionnaire exige de dépasser, voire d'abandonner, le déterminisme classique. C'est que la "position déterminée », bien qu'en partie nécessaire, a le défaut de supprimer la politique en la réduisant à une technique, que ce soit sous la forme d'un plan ou d'un programme. Ainsi, en critique du marxisme orthodoxe, il affirmera que « si les lois de l'histoire sont effectivement telles qu'à la société capitaliste ne peut succéder qu'une société communiste, il n'y a pas plus lieu de se battre pour l'avènement de celle-ci que pour le prochain lever du soleil $49 »$.

Or, le dépassement de l'illusion déterministe provenant de la nature, des lois de l'histoire ou du Parti, se réalise par et dans l'activité politique : pour « que les hommes prennent en main leurs propres affaires [...] le seul moyen [...], c'est qu'ils les prennent en main, et cela de plus en plus $5^{\circ} »$. Dire cela n'est pas une leçon de pédagogie révolutionnaire, dit-il, mais c'est mettre les individus au centre du projet: « seuls [les individus] peuvent inventer, créer une solution du problème que personne aujourd'hui ne peut soupçonner. Cela signifie qu'il faut reconnaître et laisser place à la créativité de l'histoire51. » Cette saisie de l'histoire comme création, qui accompagne la société à la fois instituée et instituante, implique à son tour de voir la politique, non pas comme l'instauration et la gestion d'un plan ou d'un programme, telle que l'implique l'idée toute contemporaine de gouvernance, mais comme "une composante de l'auto-institution de la société, la composante correspondant à un faire lucide, faire élucider autant que possible, qui vise l'institution comme telle52 ». Ainsi, la révolution n'est pas faire table rase, c'est remettre la table à sa place, voire réinventer la table; c'est, comme il le précise, "mettre en place un nouveau monde social-historique, qui est, en partie, en train de se créer, et en partie, à créer53». À cet égard, l'éthique ne suffit pas. Bien sûr, ajoute-t-il, il y a toujours une source

46 Ibid., p. 147.

47 Ibid., p. 148.

48 Ibid., p. 150.

49 Olivier Mongin et Cornelius Castoriadis, «L'exigence révolutionnaire. Entretien avec Cornelius Castoriadis », op. cit., p. 203.

50 Ibid., p. 207.

${ }^{51}$ Ibid., p. 208.

$5^{2}$ Ibid., p. 204.

53 Ibid., p. 210. 
subjective de cette adhésion qui est éthique, en ce que la subjectivité éthique est d'être responsable de ce que l'individu veut et fait, et que ce qu'il fait est fait en toute lucidité. «Nous n'inventons pas, ex nihilo, le projet révolutionnaire »: celui-ci émerge dans une société caractérisée par une crise, par une scission interne entre parties constitutives de la société. Il ne faut pas se limiter à s'opposer pour défendre notre position dans un système économique ou politique injuste, dit-il. Faire révolution nous oblige à poser le projet d'une autre société54.

Le projet révolutionnaire naît donc de ce faire qui produit la crise en se produisant lui-même : "il n'y a pas de crise objective, une société ne pourrit pas comme une poutre, il n'y a crise que dans la mesure où il y a conflit, lutte, contestation interne55 ». Les mouvements de contestation qui en émergent sont tous liés malgré leurs distinctions : « tous [...] ont une unité de signification - de relations internes entre les significations qu'ils portent : la visée d'autonomie, de l'institution d'une société autonome, c'est-à-dire l'auto-institution de la société56 ».

\section{En conclusion}

Pour Castoriadis, notre histoire a déjà connu des créations de nouvelles institutions desquelles nous pouvons tirer des exemples sans les reproduire sous la même forme.

Le projet révolutionnaire est donc présent, là, dans l'histoire effective, il parle, il se parle, ce n'est pas une « tendance objective» mais une manifestation de l'activité des hommes qui ne peut exister que si elle est, à un certain degré, consciente et qu'elle se donne sa formulation 57 .

Cette formulation advient par « le cercle de la praxis » :

Il y a une lutte et une contestation dans la société; il y a l'interprétation et l'élucidation de cette lutte (et donc de la crise) ; il y a la visée [d'autonomie] et la volonté politiques de celui qui élucide et interprète 58 .

La Commune, les Soviets, les comités de fabrique, les conseils d'usine, ont ceci de particulier qu'ils «n'ont été prédit[s], ni déduit[s] d'une théorie quelconque ; [ils] ont été créé $[s]$ par les gens, dans et par la lutte59 ». C'est en démontrant et en détruisant l'idée que le système est tout-puissant et omniscient que le faire-révolution va rendre les individus conscients qu'eux seuls ont la capacité d'accéder à l'autonomie,

\footnotetext{
54 Idem.

55 Ibid., p. 211.

56 Ibid., p. 212.

57 Ibid., p. 213.

${ }^{8}$ Idem.

59 Ibid., p. 216.
} 
« que toutes les possibilités et les capacités d'organisation de la société se trouvent en eux-mêmes ${ }^{60} »$.

Par le faire, l'histoire n'est pas déterminée, et c'est ce que démontrent deux cents ans de lutte. Il y a toujours place à la création. L'idée de la révolution créatrice de nouvelles formes de la vie sociale ne peut se faire qu'en se libérant de toute détermination par l'histoire : «L"'étonnant" [...] consiste dans l'émergence historique du projet révolutionnaire $61 »$. Castoriadis ajoute cependant à cela que toute création n'est pas nécessairement " bonne », puisque "la Raison elle-même est "obscure" ", mais c'est toutefois en posant la société comme création que nous pouvons poser la question de quelle création nous voulons et vers quoi nous voulons orienter cette création $^{62}$

Nous retiendrons que pour nous poser des questions, ce qui est déjà agir, d'un point de vue autant individuel que collectif, il faut être lucide, ne pas prendre ses fantasmes pour la réalité, s'accepter comme mortel, chercher le vrai, et donc reconnaître, comprendre et accepter les limites, nos limites, entre autres les limites à notre capacité de prévoir l'avenir.

Or, puisqu' « il n'y a pas d'existence autre qu'à plusieurs [...] la dimension sociale et historique de mon être n'en est pas une "limitation", elle en est le sol même - à partir duquel seulement des "limitations" peuvent apparaître ou ne pas apparaître63 ». Pour ainsi dire, il faut reconnaître que la liberté n'est pas sans bornes. D'ailleurs, Castoriadis soutient qu'

il n'a jamais été question pour moi de «pure liberté », ni dans le domaine de la société, ni pour ce qui est de l'individu [...] la pure liberté de l'interrogation n'est plus de la pensée, ce n'est rien du tout. [...] Si je mets tout en question, y compris le sens des mots par lesquels je mets tout en question, je ne mets plus rien en question et il n'y a plus rien 64 .

Une vraie pensée libre, dit-il, est une marche qui chaque fois doit s'appuyer sur quelque chose, se repérer aussi bien sur ce qu'elle n'est pas que sur ses « résultats précédents ». Nous revenons ainsi à sa conception de l'auto-institution explicite de la société : celle-ci n'est pas un état défini une fois pour toutes,

mais un processus continu d'auto-organisation et d'autoinstitution, la possibilité et la capacité de mettre en question les institutions et les significations instituées, de les reprendre, de les

\footnotetext{
60 Idem.

61 Ibid., p. 220.

62 Ibid., p. 222.

63 Ibid., p. 218.

64 Ibid., p. 224.
} 
transformer, d'agir à partir de ce qui est déjà là et moyennant ce qui est déjà là, mais sans s'asservir à ce qui est déjà là65.

Pour ce faire, nous n'avons pas besoin de l'État pour réaliser l'unification de la société derrière un projet révolutionnaire :

Les individus sont toujours déjà sociaux, ils n'existent, ne peuvent exister que comme toujours déjà " unifiés » dans et par une socialité en général et une socialité concrète que l' "État» luimême, là où et lorsqu'il existe, présuppose. Ce qui est toujours déjà là n'est pas assemblage physique ou biologique, ni juxtaposition de monades pensantes - mais toujours collectivité instituée comme telle, instituée par référence à des significations imaginaires qu'elle pose elle-même, parmi lesquelles se trouve toujours aussi une signification imaginaire qu'elle s'impute à elle-même. Cette fonction apparaît comme remplie par le «nous » de la collectivité considérée, mais ce "nous », bien entendu, ne reste jamais simple «nous », il est « rempli» par des références spécifiées : nous qui avons tels ancêtres, nous qui croyons à tels dieux, nous qui parlons telle langue, nous qui choisissons nos épouses de telle façon, nous qui avons subi telle cérémonie initiatique, etc. Ce sont ces références, et non pas l'« État», qui, dans une foule de sociétés, jouent le rôle de pôle unificateur'66.

Le projet révolutionnaire se résume ainsi dans la volonté et l'agir qui vise à

supprimer la hiérarchie politique, la division de la société comme division du pouvoir et du non-pouvoir. Et nous savons aussi que ce pouvoir n'est pas seulement et simplement "politique» au sens étroit; il est aussi pouvoir sur le travail et la consommation des gens, pouvoir sur les femmes, pouvoir sur les enfants, etc. Ce que nous visons, c'est l'égalité effective sur le plan du pouvoir et une société qui ait comme pôle de référence cette égalité67.

Cet agir est précédé du désir et de la volonté de transformer le monde. Toutefois, devant les enjeux auxquels doivent faire face nos sociétés si elles ne veulent pas sombrer de nouveau dans des temps obscurs, nous pouvons nous demander : avonsnous la volonté de faire le choix qui s'impose ? Car "pour qu'un véritable changement des institutions soit possible, il doit s'accompagner d'un changement correspondant des mœurs tout aussi profond 68 ». Il faut donc enrayer les facteurs contin-

65 Idem.

66 Ibid., p. 229.

67 Ibid., p. 229-230.

68 Cornelius Castoriadis, « Héritage et révolution », dans Figures du pensable. Les carrefours du labyrinthe, VI, Paris, Seuil, 1999, p. 165. 
gents qui mettent en veilleuse l'idée de révolution et qui nourrissent l'apathie des masses, qui nous voilent l'horizon des possibles. Pour ce faire, il s'agit de transformer autant les présupposés sur lesquels se fondent notre vivre en commun, nos manières de faire politique, l'organisation du travail et la définition même de celui-ci, et ce, afin de valoriser des activités qui ne sont pas productives de capital.

Nous conclurons cet excursus sur l'idée de révolution chez Castoriadis en lui laissant le dernier mot, qui résume l'ensemble de son propos :

Vouloir l'autonomie suppose de vouloir certains types d'institutions de la société et en rejeter d'autres. Mais cela implique aussi de vouloir un type d'existence historique, de rapport au passé et à l'avenir. L'un comme l'autre, le rapport au passé et le rapport à l'avenir, sont à recréer. [...] Nous devons rejeter la pseudo-modernité et la pseudo-subversion - l'idéologie de la table rase -, ainsi que l'éclectisme (le "postmodernisme») ou l'adoration servile du passé. [Dans] cet étrange « dialogue » avec le passé $[\ldots]$ nous devons reconnaître $[. .$.$] une source inépuisable$ d'altérité proche [...]. Il ne faudrait pas non plus grever notre rapport à l'avenir en lui accolant le terme fallacieux d' "utopie». Au-delà de ce que l'on appelle les possibilités du présent, dont la fascination ne peut engendrer que la répétition, nous devons, sans renoncer au jugement, oser vouloir un avenir - pas n'importe quel avenir, pas un programme arrêté, mais ce déroulement toujours imprévisible et toujours créateur, au façonnement duquel nous devons prendre part, par le travail et la lutte, pour ou contre ${ }^{69}$.

69 Ibid., p. 172-173. 